\title{
Effect of Weed and Nutrient Management on the Growth and Yield of Barley (Hordeum vulgare L.) and Associated Weeds
}

\author{
Santosh Kumar $^{1 *}$, Vivek ${ }^{1}$, N.S. Rana ${ }^{1}$, Ravindra Kumar ${ }^{2}$, R.K. Naresh ${ }^{1}$ and B.P. Dhyani ${ }^{3}$ \\ ${ }^{1}$ Department of Agronomy, ${ }^{2}$ Department of Cell Biology, ${ }^{3}$ Department of Soil Science and \\ Agricultural Chemistry, Sardar Vallabhbhai Patel University of Agriculture \& Technology \\ Meerut- 250110 (Uttar Pradesh), India \\ *Corresponding author
}

\section{A B S T R A C T}

Barley is the most important food grain crop among cereals and stands next only to rice in our country. It has significantly contributed in the success of the green revolution and has greatly helped to transform our country from a situation of ship to mouth to being setsufficient. Barley is a good supplement for nutritional requirement of human body as it

\section{Keywords}

Barley, N-PK, Vermicompost, FYM, Trisulfuron $15 \mathrm{~g}$ a.i ha ${ }^{-1}$, Yield

Article Info

Accepted:

10 January 2019

Available Online:

10 February 2019 contains $8-10 \%$ protein and $69.6 \%$ carbohydrates, $1.3 \%$ fat, $3.9 \%$ crude fiber. A field experiment was conducted during 2015-16 and 2016-17at Crop Research Center, Sardar Vallabhbhai Patel University of Agriculture and Technology, Meerut (U.P.). The experiment was laid out in split-plot design with three replications. The rabi season experiment comprised of five levels of nutrient viz.; $100 \% \mathrm{NPK}, 75 \% \mathrm{~N}-\mathrm{PK}+25 \% \mathrm{~N}$ through FYM, 75\% N-PK $+25 \% \mathrm{~N}$ through vermicompost, $50 \% \mathrm{~N}-\mathrm{PK}+50 \% \mathrm{~N}$ through FYM, 50\% N-PK $+50 \% \mathrm{~N}$ through vermicompost and four levels of weed control measures viz., Control, Two hand weeding, Trisulfuron $15 \mathrm{~g}$ a.i ha ${ }^{-1}$ and Carfentrazone ethyl $15 \mathrm{~g}$ a.i ha ${ }^{-1}$. The plant height was significantly affected at 30, 60, 90 DAS and at harvest in different treatments. The maximum plant height was recorded with the application of $75 \% \mathrm{~N}-\mathrm{PK}+25 \% \mathrm{~N}$ through vermicompost which was significantly superior to all other treatments during both years, the highest number of tillers per meter row. Lowest number of tillers was recorded in $50 \% \mathrm{~N}-\mathrm{PK}+50 \% \mathrm{~N}$ through FYM. Weed control treatments gave significantly higher yield attributes compared to control. Among the herbicides Trisulfuron $15 \mathrm{~g}$ a.i ha ${ }^{-1}$ was found more effective over Carfentrazone ethyl $15 \mathrm{~g}$ a.i $\mathrm{ha}^{-1}$. Trisulfuron $15 \mathrm{~g}$ a.i ha ${ }^{-1}$ was significantly effective and gave comparable results to two hand weeding situation. Maximum number of spikelet per spike was observed in two hand weeding and Trisulfuron $15 \mathrm{~g}$ a.i ha ${ }^{-1}$ during both years.

\section{Introduction}

Barley is a major source of food for large number of people living in the cooler semiarid areas of the world, where wheat and other cereals are less well adapted. It is a stable food of the people in the Tibet, Nepal and Bhutan. In European countries it is used only as breakfast food. The leading countries of its production are USSR, China, France, Canada, 
USA and Spain. Barley is an important cereal in India. Total area under barley in the country is about 0.7 million hectares. The chief barley growing regions in the country are higher Himalayas, central parts of eastern Uttar Pradesh, eastern parts of Rajasthan and north western parts of north Bihar. The most important uses of barley in India are as grain feed to livestock and poultry, as malt for manufacture of beer and other liquors like whisky, brandy etc. As food barley flour is used in preparing 'chapatti'. Sometimes barley is mixed with gram or wheat and then ground to flour for preparing better quantity 'chapati'. Grain is roasted and ground and used as 'sattu' (barley flour mixed in sugar and water). Grain is also broken and roughly ground into 'barley' to be used in soup. Barley is a nutritious and easily digestible cereal with 8-10\% protein, $69.6 \%$ carbohydrate, $1.3 \%$ fat, $3.9 \%$ crude fiber, $1.5 \%$ ash, $26 \mathrm{mg}$ calcium, $215 \mathrm{mg}$ phosphorus, $1.2 \%$ minerals and 336 calorific value. It is also rich in vitamin $\mathrm{B}$ complex and used to prepare dishes like chapati, sattu etc. Globally barley was cultivated on nearly 51.50 million hectare area with a production of 142.01 million metric tons. In India, during 2016-17, Barley occupied nearly 7.72 lakh hectare area producing nearly 17.26 lakh tons grain, with a productivity of $2522 \mathrm{~kg} / \mathrm{ha}$ Anonymus, (2017). The average productivity of barley in the state is far behind the attainable yielding of 40-50 q/ha Choudhary et al., (2014). Problems further aggravated by weeds compete is with the crop for moisture, nutrients, space, light etc. Moreover they increase production cost, decrease yield of the crop, harbor insects and plant diseases, decrease quality of farm produce and reduce values of the land. The weed in India are causing substantial losses to agriculture production and the annual losses in terms of money come to the Rs-1650 crores (Joshi, 2002) being higher than by insect's pests and diseases. Hence, weed control is essential for increasing barley production. It has been reported that with production of each kilogram of weed, one kilogram barley grains are reduced (Chaudhary et al., 2008). The problem of weed infestation has increased manifold as it created favourable conditions for invasion as well as luxuriant growth of weeds particularly of Phalaris minor and Avena spp. throughout barley growing area in our country (Gill et al., 1984). Therefore, cultural methods of weed control could not be performed and becomes unaffordable. Plant nutrition plays an important role in growth and productivity of a crop. As barley crop is highly responsive to applied nutrient through various sources, a proper fertility management is an important parameter for optimizing the productivity of this crop. The organic manures being cheaper and ecofriendly like FYM, compost, Vermicompost with fertilizers is receiving great attention are intensive agriculture. Application of organic along with inorganic sources not only improve soil health but with also improve the produce quality and fertilizer use efficiency and thereby reducing the cost of cultivation. Use of organic manure have been found to be promising $\mathrm{m}$ arresting the decline in productivity through correction of secondary and micronutrients deficiencies (Tripathi et al., 2010).

\section{Materials and Methods}

The present research work was carried out at the experimental field of Crop Research Center, Sardar Vallabhbhai Patel University of Agriculture and Technology, Meerut (U.P.). During 2015-16 and 2016-17at with RD-2035 variety of barley in a fourteen year old The treatments comparing of combination of five level of nutrient and four weed management option where tested in Split-Plot design with three replications, Inter Row spacing $22.50 \mathrm{~cm}$ Nutrient management 100 $\%$ NPK, $75 \%$ N-PK + $25 \%$ N through FYM, 
$75 \% \mathrm{~N}-\mathrm{PK}+25 \% \mathrm{~N}$ through vermicompost, $50 \% \mathrm{~N}-\mathrm{PK}+50 \% \mathrm{~N}$ through FYM, $50 \% \mathrm{~N}-$ $\mathrm{PK}+50 \% \mathrm{~N}$ through vermicompost and Weed management practices Control, Two hand weeding, Trisulfuron $15 \mathrm{~g}$ a.i ha ${ }^{-1}$, Carfentazone ethyl $15 \mathrm{~g}$ a.i ha ${ }^{-1}$ The experiment was conducted at (CRC) farm of the University located in Indo-Gangetic plains of Western Uttar Pradesh in Western plains zone. The farm is geographically located at $29^{0} \quad 04{ }^{\prime} \quad 19^{\prime \prime} \mathrm{N}$ latitude, $77^{0} \quad 42$ ' $50^{\prime \prime} \mathrm{E}$ longitudes and at an elevation of 237 metres above the mean sea level. Soil samples were collected to a depth of $0-15 \mathrm{~cm}$ from 10 spots in the experimental field prior to sowing of barley crop. The samples thus collected were mixed homogenously and a composite sample was drawn for analyzing various physicochemical properties. The soil of experimental site was sandy loam in texture, low in organic carbon and nitrogen and medium in available phosphorus and available potassium and neutral in reaction. Such as Organic carbon (\%) (Walkey and Black's (1947), total nitrogen $(\mathrm{kg} / \mathrm{ha}$.) by Alkaline permanganate method (Subhaiah Asija, 1956), phosphorus (kg/ha.) by Olsen's Calorimetric Method (Olsen et al., 1954), and potassium (kg/ha.) by Flame Photometric Method (Toth and Prince, 1949).

Soil $\mathrm{pH}$ and EC measured by Digital $\mathrm{pH}$ meter and conductivity meter respectively. The requisite agronomic and plant protection measures were adopted uniformly for all the treatments during the entire growing period. At maturity, data on plant characters and yield components were recorded from five randomly selected plants in each plot. The growth and yield characters were recorded such as plant height at harvest $(\mathrm{cm})$, number of total tillers plant-1, spike length $(\mathrm{cm})$, number of spike lets spike-1, number of grains spike-1, 1000-seed weight $(\mathrm{g})$, grain yield ( $t$ ha-1), straw yield ( $\mathrm{t}$ ha-1), and harvest index $(\%)$. The crop from each unit plot was harvested at full maturity to record the data on grain and straw yields. The data was analyzed statistically.

\section{Results and Discussion}

\section{Weed growth}

The most commonly surveyed weeds in the experimental situations through the two growing seasons were: Grassy weeds Wild oat (Avena ludoviciana, L.), Little seed canary-grass (Phalaris minor L.) and Nongrassy weeds Blue or Scarlet pimpernel (Anagallis arvensis L.), Common lambs quarters (Chenopodium album L.), Yellow sweet clover (Melilotus indica, L.), White sweet clover (Melilotus alba L.), Fumitory (Fumaria parviflora L.) and Sedges weeds.

\section{Effect of nutrient managements}

Nutrient and weed management practices both had significant effect on dry matter accumulation of total weeds at all the crop stages during both years. The highest total weeds dry matter accumulated where crop was grown with $50 \% \mathrm{~N}-\mathrm{PK}+50 \% \mathrm{~N}$ through FYM application during both the years (Table 1). Application of $75 \% \mathrm{~N}-\mathrm{PK}+25 \% \mathrm{~N}$ through vermicompost led to significantly reduction in total weeds dry matter accumulation irrespective of the crop growth stages during both years except at 30 DAS during 2015-16. Mean reduction against control was 27.02, 27.12 and 27.09 per cent, respectively at 30,60 and 90 DAS stages

\section{Effect of weed managements}

All the weed control measures reduced dry matter accumulation of total weeds significantly all the crop growth stage during both years (Table 1). Among the herbicides Trisulfuron $15 \mathrm{~g}$ a.i ha ${ }^{-1}$ gave significantly better control of dry matter accumulation of 
total weeds 60 and 90 DAS stages followed by Carfentrazone ethyl $15 \mathrm{~g}$ a.i ha ${ }^{-1}$ during both years. At 90 DAS the mean reduction in dry matter accumulation of total weeds was 73.13, 63.91, and 58.94 per cent, respectively with two hand weeding, Trisulfuron $15 \mathrm{~g}$ a.i $\mathrm{ha}^{-1}$, and Carfentrazone ethyl $15 \mathrm{~g}$ a.i $\mathrm{ha}^{-1}$ against control treatment. Total dry matter reduction of weeds was significant amongst the herbicidal treatment. Trisulfuron $15 \mathrm{~g}$ a.i $\mathrm{ha}^{-1}$ resulted in maximum dry matter reduction and the results obtained under these treatments. The reduction in dry matter of weeds was because of effective control of weeds by these herbicides.

Dry matter accumulation of weeds viz.; Phalaris minor, Avena ludoviciana, Chenopodium album, Angallis arvensis, Melilotus indica and others weeds were significant effect on weed control measures (Table 1). The significantly higher dry matter accumulation was found under control and it increase up to 60 DAS and their after reduction was observed. Among the herbicides Trisulfuron $15 \mathrm{~g}$ a.i ha ${ }^{-1}$ gave best control of dry matter of weeds followed by Carfentrazone ethyl $15 \mathrm{~g}$ a.i ha ${ }^{-1}$. These results are in close conformity with reports of (Singh et al., 2010).

\section{Barley growth}

\section{Effect of nutrient management}

Plant height (Table 2) increased at a faster rate during at 60 to 90 DAS was significantly affected by different nutrients treatments. The plant height was significantly affected at 30 , 60, 90 DAS and at harvest in different treatments. The maximum plant height was recorded with the application of $75 \% \mathrm{~N}-\mathrm{PK}+$ $25 \% \mathrm{~N}$ through vermicompost which was significantly superior to all other treatments during both years, except at 30 DAS. The $50 \% \mathrm{~N}-\mathrm{PK}+50 \% \mathrm{~N}$ through FYM plots resulted significant reduction in plant height than rest of the treatments at all the growth stages. Such a higher plant height in $75 \% \mathrm{~N}$ $\mathrm{PK}+25 \% \mathrm{~N}$ through vermicompost can be associated with sufficient nutrient supply at the active growth stage. Similar results of increased plant height were also reported by Ram and Mir (2006); Singh et al., (2007); Singh et al., (2008) and Singh and Pal (2011).

\section{Effect of weed managements}

Growth of barley plant in terms of plant height, number of tillers per running meter and dry matter accumulation reflects a significant improvement under different weed control treatments as compared to the control. This remarkable improvement in crop growth and development can be accounted on reduced crop weed competition. Trisulfuron $15 \mathrm{~g}$ a.i ha ${ }^{-1}$ was the best treatment for weed control during both years which resulted in maximum availability of moisture, nutrient, light and space to the crop. These results are in conformity with findings of Dixit and Singh (2008); Singh et al., (2010) and Barthwal et al., (2013).

\section{Barley yield and its attributes}

\section{Effect of nutrient management}

The number of tillers (Table 3) increased up to at 60 DAS and started declining their after at 90 DAS and at harvest. The highest number of tillers per meter row was recorded in $75 \%$ $\mathrm{N}-\mathrm{PK}+25 \% \mathrm{~N}$ through vermicompost followed by $100 \%$ NPK. Lowest number of tillers was recorded in $50 \% \mathrm{~N}-\mathrm{PK}+50 \% \mathrm{~N}$ through FYM plots at 30, 60, 90 DAS and harvest. Such a higher number of tillers in these treatments can be linked with optimum supply of essential nutrients at active tillering stage. Similar results were also reported by Pandey et al., (2009); Usadadiya and Patel (2013) and Jat et al., (2013). 
Table.1 Effect of nutrient and weed management practices on dry weight of total weed $\left(\mathrm{g} \mathrm{m}^{-2}\right)$ in barley at different stages

\begin{tabular}{|c|c|c|c|c|c|c|}
\hline \multirow[t]{3}{*}{ Treatment } & \multicolumn{6}{|c|}{ Dry weight of total weed $\left(\mathrm{g} \mathrm{m}^{-2}\right)$} \\
\hline & \multicolumn{2}{|c|}{30 DAS } & \multicolumn{2}{|c|}{60 DAS } & \multicolumn{2}{|c|}{90 DAS } \\
\hline & $2015-16$ & 2016-17 & $2015-16$ & $2016-17$ & $2015-16$ & 2016-17 \\
\hline \multicolumn{7}{|l|}{ Nutrient management } \\
\hline $100 \% \mathrm{NPK}$ & $\begin{array}{c}5.4 \\
(28.7)\end{array}$ & $\begin{array}{c}5.8 \\
(33.8)\end{array}$ & $\begin{array}{c}6.8 \\
(46.5)\end{array}$ & $\begin{array}{c}7.5 \\
(56.3)\end{array}$ & $\begin{array}{c}7.1 \\
(49.9)\end{array}$ & $\begin{array}{c}7.7 \\
(59.3)\end{array}$ \\
\hline $75 \%$ N-PK $+25 \%$ N through FYM & $\begin{array}{c}5.6 \\
(31.1)\end{array}$ & $\begin{array}{c}6.1 \\
(36.6)\end{array}$ & $\begin{array}{c}7.1 \\
(50.4)\end{array}$ & $\begin{array}{c}7.8 \\
(61.0)\end{array}$ & $\begin{array}{c}7.4 \\
(54.1)\end{array}$ & $\begin{array}{c}8.0 \\
(64.2)\end{array}$ \\
\hline $75 \% \mathrm{~N}-\mathrm{PK}+25 \% \mathrm{~N}$ through vermicompost & $\begin{array}{c}5.2 \\
(26.2)\end{array}$ & $\begin{array}{c}5.6 \\
(30.8)\end{array}$ & $\begin{array}{c}6.5 \\
(42.4)\end{array}$ & $\begin{array}{c}7.2 \\
(51.4)\end{array}$ & $\begin{array}{c}6.8 \\
(45.5)\end{array}$ & $7.4(54.1)$ \\
\hline $50 \% \mathrm{~N}-\mathrm{PK}+50 \% \mathrm{~N}$ through FYM & $\begin{array}{c}6.0 \\
(35.9)\end{array}$ & $\begin{array}{c}6.5 \\
(42.2)\end{array}$ & $\begin{array}{c}7.6 \\
(58.2)\end{array}$ & $\begin{array}{c}8.4 \\
(70.5)\end{array}$ & $\begin{array}{c}7.9 \\
(62.4)\end{array}$ & $\begin{array}{c}8.6 \\
(74.2)\end{array}$ \\
\hline $50 \% \mathrm{~N}-\mathrm{PK}+50 \% \mathrm{~N}$ through vermicompost & $\begin{array}{c}5.9 \\
(35.0)\end{array}$ & $\begin{array}{c}6.4 \\
(41.2)\end{array}$ & $\begin{array}{c}7.5 \\
(56.7)\end{array}$ & $\begin{array}{c}8.3 \\
(68.7)\end{array}$ & $\begin{array}{c}7.8 \\
(60.8)\end{array}$ & $\begin{array}{c}8.5 \\
(72.3)\end{array}$ \\
\hline $\operatorname{SEm}( \pm)$ & 0.02 & 0.02 & 0.03 & 0.04 & 0.04 & 0.04 \\
\hline C.D. $(\mathrm{P}=0.05)$ & 0.07 & 0.08 & 0.11 & 0.12 & 0.11 & 0.12 \\
\hline \multicolumn{7}{|l|}{ Weed management } \\
\hline Control & $\begin{array}{c}6.2 \\
(38.5)\end{array}$ & $\begin{array}{c}6.8 \\
(45.3)\end{array}$ & $\begin{array}{c}13.5 \\
(182.2)\end{array}$ & $\begin{array}{c}13.8 \\
(192.1)\end{array}$ & $\begin{array}{c}12.4 \\
(153.5)\end{array}$ & $\begin{array}{c}12.8 \\
(164.4)\end{array}$ \\
\hline Two hand weeding & $\begin{array}{c}4.8 \\
(22.5)\end{array}$ & $\begin{array}{c}5.2 \\
(26.5)\end{array}$ & $\begin{array}{c}6.1 \\
(36.4)\end{array}$ & $\begin{array}{c}6.7 \\
(44.1)\end{array}$ & $\begin{array}{c}6.3 \\
(39.0)\end{array}$ & $\begin{array}{c}6.8 \\
(46.4)\end{array}$ \\
\hline Trisulfuron $15 \mathrm{~g}$ a.i ha ${ }^{-1}$ & $\begin{array}{c}5.5 \\
(30.2)\end{array}$ & $\begin{array}{c}6.0 \\
(35.5)\end{array}$ & $\begin{array}{c}7.0 \\
(48.9)\end{array}$ & $\begin{array}{c}7.7 \\
(59.2)\end{array}$ & $\begin{array}{c}7.3 \\
(52.4)\end{array}$ & $\begin{array}{c}7.9 \\
(62.3)\end{array}$ \\
\hline Carfentrazone ethyl $15 \mathrm{~g}$ a.i ha ${ }^{-1}$ & $\begin{array}{c}5.9 \\
(34.4)\end{array}$ & $\begin{array}{c}6.4 \\
(40.4)\end{array}$ & $\begin{array}{c}7.5 \\
(55.7)\end{array}$ & $\begin{array}{c}8.2 \\
(67.4)\end{array}$ & $\begin{array}{c}7.7 \\
(59.6)\end{array}$ & $\begin{array}{c}8.4 \\
(70.9)\end{array}$ \\
\hline $\operatorname{SEm}( \pm)$ & 0.02 & 0.02 & 0.03 & 0.03 & 0.03 & 0.03 \\
\hline C.D. $(\mathrm{P}=0.05)$ & 0.05 & 0.06 & 0.08 & 0.09 & 0.08 & 0.09 \\
\hline
\end{tabular}

Table.2 Effect of weed management and nutrient management on plant height $(\mathrm{cm})$ of barley

\begin{tabular}{|c|c|c|c|c|c|c|c|c|}
\hline \multirow[t]{3}{*}{ Treatment } & \multicolumn{8}{|c|}{ Plant height $(\mathrm{cm})$} \\
\hline & \multicolumn{2}{|c|}{30 DAS } & \multicolumn{2}{|c|}{60 DAS } & \multicolumn{2}{|c|}{90 DAS } & \multicolumn{2}{|c|}{ At harvest } \\
\hline & $\begin{array}{c}2015- \\
16\end{array}$ & $\begin{array}{c}2016- \\
17\end{array}$ & $\begin{array}{c}2015- \\
16\end{array}$ & $\begin{array}{c}2016- \\
17\end{array}$ & $\begin{array}{c}2015- \\
16\end{array}$ & $\begin{array}{c}2016- \\
17\end{array}$ & $\begin{array}{c}2015- \\
16\end{array}$ & $\begin{array}{c}2016- \\
17\end{array}$ \\
\hline \multicolumn{9}{|l|}{ Nutrient management } \\
\hline $100 \%$ NPK & 19.80 & 17.35 & 49.45 & 43.33 & 71.81 & 58.26 & 90.04 & 85.54 \\
\hline $75 \% \mathrm{~N}-\mathrm{PK}+25 \% \mathrm{~N}$ through FYM & 18.51 & 16.22 & 46.24 & 40.54 & 67.13 & 54.43 & 85.71 & 81.43 \\
\hline $75 \% \mathrm{~N}-\mathrm{PK}+25 \% \mathrm{~N}$ through vermicompost & 21.65 & 17.79 & 55.81 & 49.00 & 80.97 & 65.57 & 93.44 & 88.77 \\
\hline $50 \% \mathrm{~N}-\mathrm{PK}+50 \% \mathrm{~N}$ through FYM & 17.65 & 15.46 & 44.09 & 38.61 & 64.04 & 51.93 & 82.27 & 78.16 \\
\hline $50 \% \mathrm{~N}-\mathrm{PK}+50 \% \mathrm{~N}$ through vermicompost & 18.28 & 16.02 & 45.67 & 40.03 & 66.31 & 53.84 & 85.63 & 81.35 \\
\hline $\operatorname{SEm}( \pm)$ & 0.34 & 0.67 & 0.95 & 0.88 & 1.34 & 0.94 & 1.68 & 1.60 \\
\hline C.D. $(P=0.05)$ & 1.12 & 1.65 & 3.08 & 2.87 & 4.37 & 3.08 & 5.47 & 5.20 \\
\hline \multicolumn{9}{|l|}{ Weed management } \\
\hline Control & 18.65 & 16.50 & 47.04 & 41.24 & 68.29 & 55.41 & 70.02 & 66.52 \\
\hline Two hand weeding & 19.71 & 17.38 & 49.53 & 43.42 & 71.91 & 58.28 & 95.19 & 90.44 \\
\hline Trisulfuron $15 \mathrm{~g}$ a.i ha ${ }^{-1}$ & 19.48 & 17.25 & 49.16 & 43.12 & 71.35 & 57.88 & 93.22 & 88.56 \\
\hline Carfentrazone ethyl $15 \mathrm{~g}$ a.i ha ${ }^{-1}$ & 18.86 & 16.59 & 47.28 & 41.44 & 68.66 & 55.65 & 91.24 & 86.68 \\
\hline $\operatorname{SEm}( \pm)$ & 0.28 & 0.52 & 0.59 & 0.58 & 0.83 & 0.70 & 1.37 & 1.30 \\
\hline C.D. $(P=0.05)$ & 0.81 & 1.30 & 1.72 & 1.68 & 2.40 & 2.01 & 3.96 & 3.76 \\
\hline
\end{tabular}


Table.3 Effect of weed management and nutrient management on number of tillers (m-1 row length) of barley

\begin{tabular}{|c|c|c|c|c|c|c|c|c|}
\hline \multirow[t]{3}{*}{ Treatment } & \multicolumn{8}{|c|}{ Number of tillers ( $\mathrm{m}^{-1}$ row length) } \\
\hline & \multicolumn{2}{|c|}{30 DAS } & \multicolumn{2}{|c|}{60 DAS } & \multicolumn{2}{|c|}{90 DAS } & \multicolumn{2}{|c|}{ At harvest } \\
\hline & $\begin{array}{l}2015- \\
16\end{array}$ & $\begin{array}{c}\text { 2016- } \\
17\end{array}$ & $\begin{array}{c}2015- \\
16\end{array}$ & $\begin{array}{c}2016- \\
17\end{array}$ & $\begin{array}{l}2015- \\
16\end{array}$ & $\begin{array}{c}2016- \\
17\end{array}$ & $\begin{array}{c}2015- \\
16\end{array}$ & $\begin{array}{c}2016- \\
17\end{array}$ \\
\hline \multicolumn{9}{|l|}{ Nutrient management } \\
\hline $100 \%$ NPK & 51.3 & 48.7 & 75.6 & 71.9 & 83.7 & 79.5 & 81.9 & 78.6 \\
\hline $75 \% \mathrm{~N}-\mathrm{PK}+25 \% \mathrm{~N}$ through FYM & 50.4 & 48.3 & 71.9 & 68.3 & 80.5 & 76.5 & 78.9 & 75.7 \\
\hline $\begin{array}{l}75 \% \mathrm{~N}-\mathrm{PK}+25 \% \mathrm{~N} \text { through } \\
\text { vermicompost }\end{array}$ & 53.5 & 50.8 & 77.6 & 73.8 & 85.8 & 81.5 & 83.8 & 80.6 \\
\hline $\mathbf{5 0 \%} \mathrm{N}-\mathrm{PK}+\mathbf{5 0 \%} \mathrm{N}$ through FYM & 46.8 & 44.5 & 68.8 & 65.4 & 76.4 & 72.5 & 74.8 & 71.8 \\
\hline $\begin{array}{l}\mathbf{5 0 \%} \mathrm{N}-\mathrm{PK}+\mathbf{5 0 \%} \mathrm{N} \text { through } \\
\text { vermicompost }\end{array}$ & 48.1 & 45.7 & 69.8 & 66.3 & 78.8 & 74.9 & 76.7 & 73.7 \\
\hline $\operatorname{SEm}( \pm)$ & 0.95 & 1.03 & 1.40 & 1.33 & 1.58 & 1.50 & 1.55 & 1.49 \\
\hline C.D. $(P=0.05)$ & 3.09 & 3.35 & 4.56 & 4.34 & 5.16 & 4.90 & 5.06 & 4.86 \\
\hline \multicolumn{9}{|l|}{ Weed management } \\
\hline Control & 41.4 & 39.5 & 58.9 & 55.9 & 56.6 & 53.7 & 54.9 & 52.7 \\
\hline Two hand weeding & 53.8 & 51.3 & 79.0 & 75.1 & 91.2 & 86.6 & 89.4 & 85.8 \\
\hline Trisulfuron $15 \mathrm{~g}$ a.i ha ${ }^{-1}$ & 52.8 & 50.2 & 77.7 & 73.8 & 89.4 & 84.9 & 87.6 & 84.1 \\
\hline Carfentrazone ethyl $15 \mathrm{~g}$ a.i ha $^{-1}$ & 51.9 & 49.4 & 75.5 & 71.7 & 86.9 & 82.6 & 85.1 & 81.7 \\
\hline $\operatorname{SEm}( \pm)$ & 0.78 & 0.76 & 1.14 & 1.08 & 1.27 & 1.21 & 1.24 & 1.19 \\
\hline C.D. $(P=0.05)$ & 2.26 & 2.21 & 3.28 & 3.12 & 3.68 & 3.49 & 3.59 & 3.45 \\
\hline
\end{tabular}

Table.4 Effect of weed management and nutrient management on spike length $(\mathrm{cm})$, no. of spikelets/spike, no. of grain/spike and test weight (g) of barley

\begin{tabular}{|c|c|c|c|c|c|c|c|c|}
\hline \multirow[t]{2}{*}{ Treatment } & \multicolumn{2}{|c|}{$\begin{array}{c}\text { Spike length } \\
(\mathbf{c m})\end{array}$} & \multicolumn{2}{|c|}{$\begin{array}{c}\text { No. of } \\
\text { spikelets/spike }\end{array}$} & \multicolumn{2}{|c|}{$\begin{array}{c}\text { No. of } \\
\text { grain/spike }\end{array}$} & \multicolumn{2}{|c|}{$\begin{array}{l}\text { Test weight } \\
\text { (g) }\end{array}$} \\
\hline & $\begin{array}{c}2015- \\
16\end{array}$ & $\begin{array}{c}2016- \\
17\end{array}$ & $\begin{array}{l}2015- \\
16\end{array}$ & $\begin{array}{c}2016- \\
17\end{array}$ & $\begin{array}{c}2015- \\
16\end{array}$ & $\begin{array}{c}2016- \\
17\end{array}$ & $\begin{array}{c}2015- \\
16\end{array}$ & $\begin{array}{c}2016- \\
17\end{array}$ \\
\hline \multicolumn{9}{|l|}{ Nutrient management } \\
\hline $100 \%$ NPK & 9.7 & 9.5 & 12.5 & 11.2 & 39.9 & 38.3 & 40.6 & 38.6 \\
\hline $75 \% \mathrm{~N}-\mathrm{PK}+25 \% \mathrm{~N}$ through FYM & 9.3 & 9.4 & 11.2 & 10.1 & 37.4 & 35.8 & 38.9 & 36.8 \\
\hline $\begin{array}{l}75 \% \mathrm{~N}-\mathrm{PK}+25 \% \mathrm{~N} \text { through } \\
\text { vermicompost }\end{array}$ & 10.5 & 10.5 & 13.7 & 12.5 & 41.9 & 39.7 & 41.4 & 38.9 \\
\hline $50 \% \mathrm{~N}-\mathrm{PK}+\mathbf{5 0 \%} \mathrm{N}$ through FYM & 8.9 & 9.1 & 9.7 & 8.9 & 35.4 & 32.9 & 37.6 & 34.7 \\
\hline $\begin{array}{l}\mathbf{5 0 \%} \mathrm{N}-\mathrm{PK}+\mathbf{5 0 \%} \mathrm{N} \text { through } \\
\text { vermicompost }\end{array}$ & 8.7 & 9.3 & 10.4 & 9.6 & 35.3 & 32.8 & 38.7 & 35.6 \\
\hline $\operatorname{SEm}( \pm)$ & 0.16 & 0.13 & 0.16 & 0.13 & $\mathbf{0 . 4 3}$ & $\mathbf{0 . 3 3}$ & 0.15 & 0.17 \\
\hline C.D. $(P=0.05)$ & $\mathbf{0 . 5 3}$ & 0.44 & 0.51 & 0.43 & 1.40 & 1.12 & $\mathbf{0 . 5 0}$ & 0.57 \\
\hline \multicolumn{9}{|l|}{ Weed management } \\
\hline Control & 7.5 & 8.3 & 9.3 & 8.4 & 34.5 & 33.7 & 36.9 & 34.6 \\
\hline Two hand weeding & 10.6 & 10.9 & 13.9 & 12.7 & 40.6 & 38.1 & 41.3 & 38.8 \\
\hline Trisulfuron $15 \mathrm{~g}$ a.i ha ${ }^{-1}$ & 10.1 & 10.0 & 12.2 & 11.1 & 39.1 & 36.5 & 40.3 & 37.6 \\
\hline Carfentrazone ethyl 15g a.i ha ${ }^{-1}$ & 9.5 & 9.1 & 10.6 & 9.7 & 37.8 & 35.3 & 39.3 & 36.7 \\
\hline $\operatorname{SEm}( \pm)$ & 0.13 & 0.18 & 0.09 & 0.08 & 0.42 & 0.34 & 0.23 & 0.25 \\
\hline C.D. $(P=0.05)$ & 0.38 & 0.54 & 0.27 & 0.25 & 1.24 & 0.98 & 0.66 & 0.71 \\
\hline
\end{tabular}


The beneficial effect of organic manures on grain, straw, biological yields and yield attributing characters might be assigned to the fact that after proper decomposition and mineralization, these manures supplied available plant nutrients directly to the plants and also had solubilising effect on fixed forms of nutrients in soil. Similar findings were also reported by (Mubarak and Singh, 2011). The combination use of organic manures and chemical fertilizers enhanced the inherent capacity of soil as reported by Pandey et al., (2009); Verma and Mathur (2009); Verma, et al., (2010) and Meena et al., (2012).

\section{Effect of weed managements}

Increase in spike length in two hand weeding was 41.6 and 31.17 per cent (Table 4) over the control during 2015-16 and 2016-17 respectively, followed by Trisulfuron $15 \mathrm{~g}$ a.i $\mathrm{ha}^{-1}$ and Carfentrazone ethyl $15 \mathrm{~g}$ a.i ha ${ }^{-1}$ was significantly higher over control.

Maximum number of spikelet per spike was observed in two hand weeding 13.97 and 12.67 during both years followed by Trisulfuron $15 \mathrm{~g}$ a.i ha ${ }^{-1}$ during 2015-16 and 2016-17. Highest grains per spike and test weight was observed in two hand weeding 40.60 and 38.12 and 41.33 and $38.79 \mathrm{~g}$ followed by Trisulfuron $15 \mathrm{~g}$ a.i ha $\mathrm{ha}^{-1}$ and significantly superior to control during both years. Similar finding were also reported by Chopra et al., (2008). The harvest index was significantly higher in two hand weeding (42.06 and 39.46) followed by Trisulfuron $15 \mathrm{~g}$ a.i ha ${ }^{-1}$ and Carfentrazone ethyl $15 \mathrm{~g}$ a.i $\mathrm{ha}^{-1}$ during 2015-16 and 2016-17. The better performance of these treatments in terms of yield could be attributes to better expression of their yield attributes due to reduction in crop weed competition. This could be attributed to the selectivity of these herbicides to crop and significantly effect on weeds has already been reported Dixit and Singh (2008).
It is concluded that the adoption of $75 \% \mathrm{~N}$ $\mathrm{PK}+25 \% \mathrm{~N}$ through vermicompost significantly improved the growth, yield attributing characters, yield and productivity of barley crop. Application of $100 \%$ NPK gave at par results for these parameters. Among the weed control practices Trisulfuron $15 \mathrm{~g}$ a.i ha ${ }^{-1}$ improved the different growth parameters and yield of barley crop over the control.

\section{Acknowledgement}

Studentship support, provided by Department of Agronomy, college of Agricultural, Sardar Vallabhbhai Patel University of Agriculture \& Technology Meerut to conduct present research work as part of Ph.D (Agronomy) thesis, is duly acknowledged.

\section{References}

Anonymous (2016-17) Agricultural Statistics Division. New Delhi.

Barthwal, A., Bhardwaj, A.K., Chaturvedi, S. and Pandiaraj, T. 2013. Site specific NPK recommendation in wheat (Triticum aestivum) for sustained crop and soil productivity in mollisols of Tarai region. Indian Journal of Agronomy 58 (2): 208-214.

Chopra, N.K., Chopra, N. and Sinha 2008. Influence of new broadleaf herbicides on weed control, seed yield and quality of some wheat (Triticum aestivum) cultivars. Indian Journal of Agriculture Science 78 (5): 405-407.

Chaudhary, S.U., M. Hussain, M.A. Ali and J. Iqbal. 2008. Effect of weed competition period on yield and yield components of wheat Indian Journal of Agriculture research.. 46: 47-53.

Choudhary, S., Yadav, L.R., Shivran, A.C. and Puniya, M.M. (2014) Effect of fertilizers and manures with foliar application of iron on barley (Hordeum 
vulgare L.). Green Farming, 5 (1): 41 45

Dixit, A. and Singh, V.P. 2008. Efficacy of a ready mix application of carfentrazone plus, isoproturon (affinity) to control weed in wheat (Triticum aestivum). Indian Journal of Agriculture Science 78:6 495-497.

Gill, H.S., Singh, Ajmer and Walia, U.S. 1984. Tolerance of weed cultivars to metoxuron. Indian Journal of weed science 16: 176-181.

Jat, G., Majumdar, Jat, S.P., N.K. and Majundar, S.P. 2013. Potassium and zinc fertilization of wheat (Triticum aestvum) in Western arid zone of India. Indian Journal of Agronomy 58 (1) $67-$ 71.

Joshi, N. C.2002. "Manual of weed control". Research publication 7615-B, East Azad nagar, Delhi-110 051.

Meena, B.L., Phogat, B.S., Jat, S.L., Singh, A.K. and Sharma, H.B. 2012. Effects of planting and integrated nutrient management systems on root phenology and grain yield of wheat. Etennded Summaries: $3^{\text {rd }}$ International Agronomy Congress, Nov. 26-30, New Delhi, India.

Pandey, I.B., Dwivedi, D.K. and Pandey, R.K. 2009. Integrated nutrient management for sustaining wheat (Triticum aestivum) production under late sown condition. Indian Journal of Agronomy 54 (3): 306-309.

Ram T. and Mir, M.S. 2006. Effect of integrated nutrient management on yield and yield attributing characters of wheat (Triticum aestivum). Indian Journal of Agronomy 31 (3): 189-192.

Singh, R.K., Singh, S.K. and Singh, L.B. 2007. Integrated nitrogen management in wheat (Triticum aestivum). Indian Journal of Agronomy 52 (2): 124-126.

Singh, F., Kumar, R. and Pal, S. 2008. Integrated nutrient management in rice- wheat cropping system for sustainable productivity. Journal of the Indian Society of Soil Science vol. 56, No.2, pp 205-208.

Singh, Samunder, Ashok, Yadav, S.S. Punia, R.S. Malik and R.S. Balyan 2010. Interaction of stage of application and herbicides on some Phalaris minor populations. Indian Journal of Weed Science 42: 144-154.

Singh, Samunder, Ashok, Yadav, S.S. Punia, R.S. Malik and R.S. Balyan 2010. Interaction of stage of application and herbicides on some Phalaris minor populations. Indian Journal of Weed Science 42: 144-154.

Singh, Samunder., S.S. Punia., Ashok Yadav and V.S. Hooda 2011. Evaluation of carfentrazone-ethyl+metsulfuronmethyl against broadleaf weeds of wheat. Indian Journal of Weed Science 43 (1\&2): 12-22.

Subbiah, B.V. and Asija, G.L. 1956. A rapid procedure for the estimation of available Nitrogen in soil, curr. Sci 25:255-260.

Toth, S.J., and Prince, A.L. 1949. Estimation of cation exchange capacity and exchangeable $\mathrm{Ca}, \mathrm{K}$ and $\mathrm{Na}$ contains of soil by Flame Photometer. Soil Sci., 67:430-445.

Tripathi, M.K., Chaturvedi, S., Shukla, D.K. and Mahapatra, B.S. 2010.yield performance and quality in Indian mustard (Brassica juncia) as affected by integrated nutrient management. Indian Journal of Agronomy 55 (2): 138-142.

Usadadiya, V.P. and Patel, R.H. 2013. Influence of preceding crops and nutrient management on productivity of wheat (Triticum aestivum)-based cropping system. Indian Journal of Agronomy 85 (1): 15-18.

Verma, G. and Mathur, A.K. 2009. Effect of integrated nutrient management on active pools of soil organic matter under 
maize-wheat system of a Typic Haplustept. Journal of the Indian Society of Soil Science 57 (3) pp 317322.

Verma, G., Mathur, A.K., Bhandari, S.C. and Kanthaliya, P.C. 2010. Long term effect of integrated nutrient management on properties of a typichaplustept under maize-wheat cropping system.Journal of the Indian society of soil science, vol, 58(3): 299-302.

Walkey, A. and Black, I.A. 1934. An examination of the digital jar if method/determining soil organic matter and a proposal modification of the Chromic Titration Method. Soil Sci., 37:29-38.

\section{How to cite this article:}

Santosh Kumar, Vivek, N.S. Rana, Ravindra Kumar, R.K. Naresh and Dhyani, B.P. 2019. Effect of Weed and Nutrient Management on the Growth and Yield of Barley (Hordeum vulgare L.) and Associated Weeds. Int.J.Curr.Microbiol.App.Sci. 8(02): 993-1001. doi: https://doi.org/10.20546/ijcmas.2019.802.115 